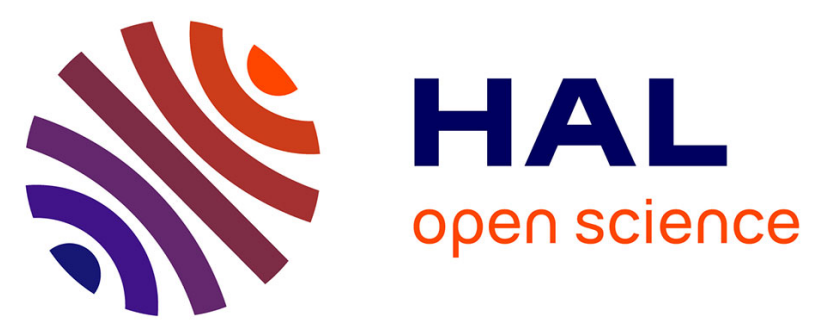

\title{
New feature in selective reflection with a highly parallel window: phase-tunable homodyne detection of the radiated atomic field
}

\author{
Aram V. Papoyan, G. G. Grygoryan, S. V. Shmavonyan, David Sarkisyan, \\ Jocelyne Guéna, Michel Lintz, Marie-Anne Bouchiat
}

\section{To cite this version:}

Aram V. Papoyan, G. G. Grygoryan, S. V. Shmavonyan, David Sarkisyan, Jocelyne Guéna, et al.. New feature in selective reflection with a highly parallel window: phase-tunable homodyne detection of the radiated atomic field. The European Physical Journal D : Atomic, molecular, optical and plasma physics, 2004, 30, pp.265. 10.1140/epjd/e2004-00088-0 . hal-00002780

\section{HAL Id: hal-00002780 \\ https://hal.science/hal-00002780}

Submitted on 6 Sep 2004

HAL is a multi-disciplinary open access archive for the deposit and dissemination of scientific research documents, whether they are published or not. The documents may come from teaching and research institutions in France or abroad, or from public or private research centers.
L'archive ouverte pluridisciplinaire HAL, est destinée au dépôt et à la diffusion de documents scientifiques de niveau recherche, publiés ou non, émanant des établissements d'enseignement et de recherche français ou étrangers, des laboratoires publics ou privés. 


\title{
New feature in selective reflection with a highly parallel window: phase-tunable homodyne detection of the radiated atomic field
} Short title: Phase tunable homodyne detection in selective reflection

\author{
A.V. Papoyan, G.G. Grigoryan, S.V. Shmavonyan, D. Sarkisyan, \\ Institute for Physical Research, NAS of Armenia, Ashtarak-2, 378410 Armenia \\ J. Guéna, M. Lintz, M.A. Bouchiat \\ Laboratoire Kastler Brossel $l^{\dagger}$ et Fédération de Recherche ${ }^{\ddagger}$ \\ Département de Physique de l'Ecole Normale Supérieure, \\ 24 rue Lhomond, 75231 Paris Cedex 05, France
}

31 March 2004

\begin{abstract}
Selective reflection (SR) of light from a cesium vapor - sapphire interface close to the $\mathrm{D}_{2}$ resonance line has been studied by use of a sapphire window with highly parallel surfaces. Temperature-tuning of the Fabry-Perot behaviour of the window [Jahier et al., Appl. Phys. B 71, 561 (2000)], resulting in a change of 0.5 to $26 \%$ of the window reflection coefficient, dramatically affects both the magnitude and lineshape of the SR resonant atomic signal. For nearly zero window reflection, the case of particular interest, the absorptive properties of the atomic medium govern the signal shape, as opposed to the usual dispersive ones in the "ordinary" SR. This is a manifestation of homodyne detection of the radiated atomic field. The numerical simulation based on a model, which accounts for all the processes involved, shows a good agreement with the experimental spectra. Possible applications for laser and atomic spectroscopy, in particular tunable locking of laser frequency, are discussed.

PACS numbers: 39.30.+w: Spectroscopic techniques; 42.25.Gy: Edge and boundary effects; reflection and refraction; 32.70.Jz: Line shapes, widths, and shifts; 42.25.Hz: Interference
\end{abstract}

\section{Introduction}

The selective reflection (SR) technique $[1,2,3,4]$ is known to be a powerful tool for a number of applications in atomic spectroscopy and laser physics. Being free of complications imposed by propagation and inhomogeneous broadening effects, SR allows one to study the response of a thin atomic vapor layer (the thickness is of the order of the wavelength, or less) behind the entrance window of an optical cell, over a wide range of atomic vapor densities from $N_{a t} \cong 10^{12} \mathrm{~cm}^{-3}$ to $\cong 10^{19} \mathrm{~cm}^{-3}$ [5]. Among the applications of SR spectroscopy are: determination of homogeneous broadening, in particular collisional self-broadening

\footnotetext{
${ }^{*}$ Corresponding author. E-mail: guena@lkb.ens.fr, Fax: +33-1-44323434, Tel: +33-1-44323596

${ }^{\dagger}$ Laboratoire de l'Université Pierre et Marie Curie et de l'ENS, associé au CNRS (UMR 8552)

${ }^{\ddagger}$ Fédération de Recherche de l'ENS, associée au CNRS (FR684)
} 
and shift of atomic resonance lines [6,7]; study of the van der Waals interaction of atoms with dielectric surfaces [8]; study of coherent and magneto-optical processes [9, 10, 11]; locking a diode laser frequency to atomic resonance lines [12, 13], etc.

In most cases, the optical response in selective reflection is associated with dispersive properties of the atomic vapor. Theoretical descriptions of lineshapes encountered in SR spectroscopy under various conditions and/or approximations have been given by several authors $[4,14,15,16]$. Nevertheless, there are a few publications where more complex vapor-dielectric interfaces have been studied, namely with an intermediate thin metallic layer [17] or an internal dielectric coating [18]. In those cases, the signal mixes absorptive and dispersive components of the effective resonant susceptibility, and one can derive conditions under which the lineshape depends only on the imaginary part of the vapor admittance at the boundary.

In [19], a cell with highly parallel sapphire windows has been shown to allow temperature tuning of the reflection losses at the windows, which range from $26 \%$ per window down to nearly zero, depending on the window temperature, and hence on the interference order of the Fabry-Perot etalon behavior of the window.

In the present paper we report on the first observation of the selective reflection obtained with a temperaturetunable cell containing an atomic cesium vapor, along with a theoretical model, which describes experimental spectra and their temperature dependence. We study how the lineshape of the reflected signal changes with the window temperature and ask whether it is possible to distinguish in this signal absorptive and dispersive contributions of the atomic vapor response. Besides scientific interest, this question is of particular relevance for the practical implementation of (frequency-tunable) locking of laser frequency to atomic resonance lines.

\section{Experimental, measurements and results}

We used an $80 \mathrm{~mm}$-long sealed-off alumina cell, with a $10 \mathrm{~mm}$ inner diameter, closed by $d=0.5 \mathrm{~mm}$ thickness sapphire windows with a crystalline orientation (0001) to better than 15 arc minutes ${ }^{1}$. Both the tilt angle between the two surfaces of the windows $(<10 \mu \mathrm{rad})$ and the surface roughness $(<\lambda / 10)$ meet requirements for temperature-tunable windows listed in [19]. The cell was placed in a two-section oven, which allowed us to control and stabilize (to better than $\pm 0.5{ }^{\circ} \mathrm{C}$ accuracy) the temperatures of the cell body and of the attached side arm by means of an active microprocessor feedback system. Throughout the experiment, the side arm which contains a small amount of metallic Cs which determines the saturated vapor density, was kept at $T_{s a}=160^{\circ} \mathrm{C}$. The corresponding number density of Cs atoms is $N_{C s} \cong 2.9 \times 10^{14} \mathrm{~cm}^{-3}$ (the precise value depends on the window temperature, which was varied in the range $T_{w}=190-232{ }^{\circ} \mathrm{C}$ ). Note that in this temperature regime a noticeable collisional self-broadening of the $\mathrm{Cs}_{2} \mathrm{D}_{2}$ line takes place ( $\gamma_{c o l} \cong 33 \mathrm{MHz}$ [6]). The choice of a relatively high vapor pressure was justified by the necessity of direct recording of the SR spectra with a high signal-to-noise ratio despite the absence of frequency modulation.

A standard grating external cavity diode laser $\left(P_{L}=30 \mathrm{~mW} ; \lambda=852 \mathrm{~nm} ; \Delta \nu_{L} \sim 3 \mathrm{MHz}\right)$ was used as a tunable radiation source in the region of the $\mathrm{D}_{2}$ line of atomic cesium. A fraction of the laser radiation was sent to an auxiliary $\mathrm{Cs}$ saturated absorption (SA) setup to provide a frequency reference. The main $\oslash=0.5 \mathrm{~mm}$ linearly polarized radiation beam was directed under near-normal incidence (angle $\simeq$ $7 \mathrm{mrad}$ ) onto the front window of the Cs cell. The power of the incident beam $P_{\text {inc }}$ was attenuated to 20

\footnotetext{
${ }^{1}$ Meller Optics Inc., 120 Corliss St., Providence, RI 02904, USA
} 
$\mu \mathrm{W}$ to reduce saturation and optical pumping effects. The reflected radiation power $P_{\text {refl }}$ was detected by a photodiode placed $40 \mathrm{~cm}$ away from the window. The small detection solid angle $\left(5 \times 10^{-5} \mathrm{srad}\right)$ allowed us to suppress the contribution of resonance fluorescence to the recorded signal. The photodiode was followed by an operational amplifier and a two-channel digital oscilloscope for simultaneous recording of SR and SA spectra. To obtain SR spectra, the laser radiation frequency was linearly scanned by up to $\Delta \nu_{\text {scan }}=3.5$ $\mathrm{GHz}$ through the spectral region covering the $F_{g}=3 \rightarrow F_{e}=2,3,4$ hyperfine transitions of the $\mathrm{Cs}_{2}$ line. The scanning was realized by application of periodical triangular ramps with $24 \mathrm{~ms}$ rise/fall time. The selective reflection measurements, which were performed with $T_{s a}=160{ }^{\circ} \mathrm{C}$ while $T_{w}$ was varied from 190 ${ }^{\circ} \mathrm{C}$ to $232{ }^{\circ} \mathrm{C}$ by step of $1{ }^{\circ} \mathrm{C}$, are shown in Fig.1. The raw spectra are presented in Fig.1 $a$. One can see that the SR signal is superimposed upon the window "background" reflection, strongly variable with the window temperature. The magnitude of this off-resonance contribution is obtained using the equations presented in [19]:

$$
R(\varphi)=\frac{P_{r e f l}}{P_{\text {inc }}}=\frac{1}{1+\left(\frac{2 n}{\left(n^{2}-1\right) \sin \varphi}\right)^{2}}
$$

where the refractive index of sapphire $n=1.759$, and $\varphi$ is the phase acquired after an internal reflection:

$$
\varphi(T)=\varphi\left(T_{0}\right)+\frac{2 \pi d}{\lambda}\left(\frac{\partial n}{\partial T}+\alpha_{T} n\right)\left(T-T_{0}\right)
$$

where $\alpha_{T}=7 \times 10^{-6} \mathrm{~K}^{-1}$ is the linear expansion coefficient and $\frac{\partial n}{\partial T}=15 \times 10^{-6} \mathrm{~K}^{-1}$ the temperature coefficient for sapphire ${ }^{2}$. From Eqs.(1) and (2), the off-resonance reflection coefficient must vary from $R_{\min }=0$ to $R_{\max }=26.2 \%$ in a temperature interval $\Delta T=15.6{ }^{\circ} \mathrm{C}$, which is in good agreement with the experiment. One can see that the atomic resonant contribution to the reflected signal (triple peak/dip structure) is weak. To make this contribution visible, we subtracted in Fig. $1 b$ the off-resonance reflection (taken as the mid-value of $\mathrm{R}$ at the edges of the scanning region) from the total signal. Note that even after this procedure, an overall temperature-dependent wavy pattern remains. The latter is due to the phase dependence on the radiation frequency at fixed temperature:

$$
\varphi(\nu)=\varphi\left(\nu_{0}\right)+\frac{2 \pi n d}{c}\left(\nu-\nu_{0}\right)
$$

For the $900 \mathrm{MHz}$-frequency region covered in Fig.1, the reflection slope changes from $\cong 0.01 \% / \mathrm{GHz}$ for $\varphi \cong m \pi / 2$ to $\cong 0.47 \% / \mathrm{GHz}$ for $\varphi \cong(2 m+1) \pi / 4$ ( $m$ integer).

To eliminate better the contribution from the off-resonance reflection and to visualize the change of the SR (resonant) lineshape with the window temperature, we present in Fig.1c the frequency derivatives of the direct reflection spectra depicted in Fig. $1 a$. Note that under the conditions of our experiment $(10,000$ acquired measurement points per scan, fast response of the photodetector), this procedure is equivalent, to some extent, to the application of a frequency modulation with subsequent synchronous detection (except for noise rejection). As can be seen from Fig. $1 c$, a flip of the derivative occurs every $\cong 16{ }^{\circ} \mathrm{C}$, at the window temperatures for which the phase $\varphi$ passes through $m \pi$ (reflection minima) or $(2 m+1) \pi / 2$ (reflection maxima).

\footnotetext{
${ }^{2}$ This coefficient actually depends on the method used for the crystal growth.
} 
In Fig. $2 a$, the measured SR response is shown for the window temperatures which correspond to the phase values $\varphi=0, \pi / 4, \pi / 2$, and $3 \pi / 4$ (left column). The evolution of the SR spectral lineshape with the window temperature is best seen on the signal derivatives presented along with the original spectra (right and left graphs, respectively). Obviously, the shape of a spectrum changes with the window temperature. For comparison with the case of an "ordinary" selective reflection (with no interference effects involved), the lower graph in Fig. $2 a$ shows a SR spectrum, and its frequency derivative, taken in similar temperature conditions $^{3}$ but with the use of a vapor cell fitted with a wedged sapphire window. Since this measurement has been performed on a different setup, with a free running laser diode $\left(\Delta \nu_{L} \cong 25 \mathrm{MHz}\right)$, direct comparison of the signal magnitudes is not relevant. However, the comparison of the spectral lineshapes for these two cases holds justified. One can see that the lineshape of the ordinary SR signal is similar to the one of the $T_{w}=211^{\circ} \mathrm{C}$ signal where reflection maximum occurs, though with less pronounced sub-Doppler features. The most substantial variation of the signal lineshape occurs in the temperature region where the off-resonance reflection minimum takes place. This region of particular interest $\left(190-198^{\circ} \mathrm{C}\right)$ is explored in more detail in Fig. $3 a$. As can be seen from these graphs, indeed, a variation of the window temperature by only $1{ }^{\circ} \mathrm{C}$ has a dramatic effect upon the reflection signal line shape, while in the other temperature regions the variation of the reflection signal with $T_{w}$ is less significant.

The measurements were also performed at an elevated side arm temperature $T_{s a}=180{ }^{\circ} \mathrm{C}$ (i.e., with an increased Cs vapor density $N_{C s}=6.8 \times 10^{14} \mathrm{~cm}^{-3}$ ) to enhance the atomic signal (albeit at the expense of an increased collisional linewidth $\gamma_{c o l} \cong 78 \mathrm{MHz}$ ). The spectra (not shown) are not qualitatively different from those recorded at $T_{s a}=160{ }^{\circ} \mathrm{C}$. Special checks of a possible dependence of the reflection spectra on the incident laser radiation polarization (linear, vertical or horizontal; elliptical), for various window temperature ranges, have shown that the spectra are insensitive to changes of laser polarization.

Obviously temperature changes of the window can affect dramatically the SR spectra, while it has no significant consequence on the way the atoms interact with the light field. Indeed, this reveals the homodyne beating between the non-resonant reflected field and the emitted resonant field, which bears the atomic response. The phase of the non-resonant reflected field changes depending on the interference regime, so that the total reflected intensity contains a predominantly dispersive or absorptive contribution, depending on the interference order, and hence on the window temperature. This issue is discussed in the next section.

\section{Model and discussion}

Our aim here is to calculate the amplitude of the total electric field $E_{R}$ backreflected from the Fabry-Perot constituted by the window, taking into account the backward radiation field generated in the resonant vapor (see Fig.4 for the geometry):

$$
E_{R}=E_{R(n r)}+E_{R(a t)}
$$

where $E_{R(n r)}$ is the "non-resonant" contribution that would arise in an empty cell (with no resonant vapor), and $E_{R(a t)}$ the "atomic" contribution caused by the resonant response of the atomic vapor.

To this purpose, following the lines of [20], we use :

\footnotetext{
${ }^{3}$ For this case, the value of the window temperature is of secondary importance: a change of $T_{w}$ by $10{ }^{\circ} \mathrm{C}$ alters $N_{C s}$ by less than $2 \%$.
} 
1) the continuity equations for the radiation field at each interface, denoting $r_{i j}$ and $t_{i j}$ the amplitude coefficients of reflection and transmission, respectively, at the interface between the $i$-th to $j$-th media

$$
r_{i j}=\frac{n_{i}-n_{j}}{n_{j}+n_{i}} ; \quad t_{i j}=1+r_{i j}=\frac{2 n_{i}}{n_{j}+n_{i}}
$$

(in our case $n_{1}=n_{3}=1$ and $n_{2}=\mathrm{n}$, the refractive index of the dielectric window); and

2) the Maxwell equations for the propagation of the backward atomic field through the resonant vapor, without using the slowly varying envelope approximation.

Assuming that the beam is completely absorbed in the vapor (cell length $\mathrm{L} \gg$ absorption length), we obtain for the total reflected field

$$
E_{R}=r_{12} E_{0}+\frac{t_{12} t_{21} r_{23} E_{0} \exp (2 i \varphi)}{1-r_{23} r_{21} \exp (2 i \varphi)}+\frac{t_{21} t_{32} E_{a t} \exp (i \varphi)}{1-r_{23} r_{21} \exp (2 i \varphi)}
$$

where $\varphi=\frac{2 \pi n_{2} d}{\lambda}$ is the phase acquired after a single passage through the dielectric sapphire plate ${ }^{4}$.

The first two terms correspond to the non-resonant contribution $E_{R(n r)}$. With $R(\varphi)=\left|\frac{E_{R(n r)}}{E_{0}}\right|^{2}$, one recovers the usual expression (Eq. (1)) for the intensity reflection coefficient by an ordinary parallel plate.

The resonant atomic contribution is given by the last term in Eq. (6)

$$
E_{R(a t)}=\frac{t_{21} t_{32} E_{a t} \exp (i \varphi)}{1-r_{23} r_{21} \exp (2 i \varphi)}
$$

which involves the backward atomic field generated in the vapor

$$
E_{a t}=2 i k \int_{0}^{L=\infty} P(x) \exp (2 i k x) d x,
$$

where $P(x)$ is the atomic polarization and $k=\omega / c$. We define the atomic response $\Im_{s}$ by rewriting

$$
E_{a t}=\frac{t_{12} t_{23} E_{0} \exp (i \varphi)}{1-r_{23} r_{21} \exp (2 i \varphi)} \Im_{s} .
$$

where the input field transmitted in the vapor after multiple reflections through the window is singled out.

As in previous works $[10,15,18,20]$, we assume a linear approximation in the light field and neglect the back reaction of the atomic polarization on the light field that drives the atoms. Solving the density matrix equations relevant to the transitions involved, we obtain

$$
\Im_{s}=\frac{N_{C s}}{\hbar \sqrt{\pi}} \sum_{k=1}^{3} \frac{\left|d_{k}\right|^{2}}{\Gamma_{D}} \int_{0}^{\infty} \frac{\exp \left(-x^{2}\right) d x}{\frac{\left(\omega-\omega_{k}\right)}{\Gamma_{D}}+x+\frac{i \Gamma}{\Gamma_{D}}-\frac{\Delta \omega_{l}}{\Gamma_{D}}} .
$$

Here the indices $k=1,2,3$ account for the three hyperfine transitions $\left(F_{g}=3 \rightarrow F_{e}=2,3,4\right)$ with resonance frequencies $\omega_{k}$ and transition dipole moments $d_{k}$ [21]; $\Gamma_{D}=u_{0} / \lambda$ is the Doppler width ( $u_{0}$ is the mean atomic velocity), $\Gamma_{k}=\gamma+\gamma_{c o l}+\Delta \nu_{L}$ the total homogeneous width including natural linewidth, collisional broadening, and laser linewidth, correspondingly, and $\Delta \omega_{l k}$ the Lorentz redshift, taken as $-\gamma_{c o l} / 3$ [15], [22].

\footnotetext{
${ }^{4}$ Tuning of $\varphi$ with the window temperature is described by Eq. (2).
} 
Inserting Eq. (9) into Eq. (6), we obtain the overall intensity reflection coefficient $R$ :

$$
R=\left|\frac{E_{R}}{E_{0}}\right|^{2}=\left|r_{12}+\frac{t_{12} t_{21} \exp (2 i \varphi)}{1-r_{23} r_{21} \exp (2 i \varphi)}\left(r_{23}+\frac{t_{23} t_{32} \Im_{s}}{1-r_{23} r_{21} \exp (2 i \varphi)}\right)\right|^{2} .
$$

Using this equation with $n_{1}=n_{2}=n$ and $n_{3}=1$, one recovers the reflection coefficient for the case of "ordinary" SR at the 2-3 boundary:

$$
R_{\text {ordinary }}=\left|r_{23}+t_{23} t_{32} \Im_{s}\right|^{2} .
$$

In the linear approximation in $\Im_{s}$, it involves only the dispersive contribution of the atomic response: $R_{\text {ordinary }} \cong r_{23}^{2}+2 r_{23} t_{23} t_{32} \operatorname{Re}\left\{\Im_{s}\right\}$.

In our case of the parallel plate $\left(n_{1}=1, n_{2}=n, n_{3}=1\right)$ we find

$$
R=\frac{\sin ^{2} \varphi}{\alpha^{2}+\sin ^{2} \varphi}+\alpha^{2} \frac{2 \sin \varphi\left[\beta \sin \varphi \operatorname{Re}\left\{\Im_{s}\right\}+\alpha \cos \varphi \operatorname{Im}\left\{\Im_{s}\right\}\right]+\alpha^{2}\left|\Im_{s}\right|^{2}}{\left(\alpha^{2}+\sin ^{2} \varphi\right)^{2}},
$$

where $\alpha=\frac{r_{12}^{2}-1}{2 r_{12}}=\frac{2 n}{n^{2}-1}$ and $\beta=\frac{n^{2}+1}{n^{2}-1}$. Note that the last (quadratic) term in the numerator of Eq.(13) is not omitted, since it yields a non-negligible contribution in the vicinity of $\varphi=m \pi$. The reflection coefficient $R$ as given by Eq. (13) depends not only on $\operatorname{Re}\left\{\Im_{s}\right\}$ (dispersive contribution), as in "ordinary" SR, but also on $\operatorname{Im}\left\{\Im_{s}\right\}$ (absorptive contribution). Depending on the phase $\varphi$, these terms may happen to be of comparable magnitude. Near the reflection minima $(\sin \varphi \ll 1)$ the $\cos \varphi \operatorname{Im}\left(\Im_{s}\right)$ term dominates the $\sin \varphi \operatorname{Re}\left(\Im_{s}\right)$ term and it accounts for the change of sign of the resonant signal observed for a positive or negative variation of temperature (Fig. $3 a$ ). In fact, though we record the reflection intensity, usually supposed to be "sign-insensitive", the implementation of the temperature-tunable Fabry-Perot window allows us to realize homodyne detection of the atomic signal in selective reflection. With respect to "ordinary" $\mathrm{SR}$, a noticeable difference appears close to the minima of the window reflection depending on whether the phase of the reflected field adds or subtracts to the phase of the radiated atomic field.

We performed the numerical simulation of the reflected signal using Eq. (11) (and Eq. (12) for ordinary SR) for the experimental conditions of Figs. $2 a$ and $3 a$, with the phase values that correspond to the given window temperatures, without any fitted parameter. The results shown in Figs. $2 b$ and $3 b$ are in rather good agreement with the experimental results (both for the SR spectra and their derivative). We ascribe minor mismatches, most noticeable on the wings of the reflection spectra, to the slight frequency dependence of the laser intensity and other experimental imperfections, as well as to the fact that the laser frequency dependence of the non-resonant reflection (see Section 2) has not been included in the model. This agreement tends to support the model we have proposed.

\section{Possible application for the laser frequency locking}

As mentioned in the introduction, selective reflection techniques can be used for locking a laser frequency to atomic resonance lines $[12,13]$. The practical usefulness of the locking technique increases if the locked frequency can be tuned within some limits around the resonance line. In [13], a $\sim 5 \mathrm{MHz}$ tuning of the servo-lock point by means of variation of the vapor pressure has been reported. Extension of this range to $\sim 300 \mathrm{MHz}$ is possible with the use of magneto-optical methods [23, 24, 25], but this requires implementing a precise polarization technique and application of a variable magnetic field. 
The substantial variation of the line shape of the SR signal with the window temperature discussed above can be exploited for the realization of frequency-tunable locking of the laser diode frequency to the atomic hyperfine transitions. To prove this statement, we have performed an analysis based on the model of Section 3.

Standard frequency locking techniques require frequency modulation of the laser radiation with lock-in detection of the atomic optical response signal. The sign and the amplitude of the error signal generated in the feedback scheme depend on the deviation of the actual frequency from the zero-crossing points of the frequency derivative of the optical spectrum (dispersive-shaped locking signal). In Fig. 5, we present the dependence of the frequency positions of these zero-crossing points on the acquired reflection phase for the conditions of the present experiment (see Section 2). There are six such points for the group of the three hyperfine transitions $F_{g}=3 \rightarrow F_{e}=2,3,4$, three on the falling slopes and three on the rising slopes of the derivative signal (left and right graphs in Fig.5, respectively). As can be seen from the left graphs, a variation of the window temperature by $35^{\circ} \mathrm{C}$, which corresponds to the phase variation $\Delta \varphi=\pi$, changes the zero-crossing points on the falling edge by $\pm 30-40 \mathrm{MHz}$ around each hyperfine transition. At the same time, the zero-crossing points on the rising edge undergo some change between neighbouring hyperfine transitions, and a broad ( $>600 \mathrm{MHz}$ ) deviation on the red wing of the $F_{g}=3 \rightarrow F_{e}=2$ transition. Of course, the range of tuning depends generally on the particular parameters of the experiment (chosen optical transitions, vapor density, window material and thickness, etc.

As final remark, we point out the relative simplicity of the proposed tunable locking method where only a control of the window temperature is required for tuning the frequency with the averaged slope coefficient of $2 \mathrm{MHz} /{ }^{\circ} \mathrm{C}$. We note that temperature control units with $<0.1{ }^{\circ} \mathrm{C}$ accuracy are commercially available. The practical realization of the locking technique however, lies beyond the scope of the present work.

\section{Conclusion}

We have studied selective reflection of light from the dielectric window-resonant atomic vapor interface, both experimentally and theoretically, exploiting the Fabry-Perot behavior of a sapphire window with highly parallel faces. Variation of the window temperature by $\Delta T_{w} \cong 35{ }^{\circ} \mathrm{C}$ results in a full-range variation of the reflection phase. Depending on this phase, the lineshape and magnitude of the atomic signal change dramatically. The greatest variation of the reflection spectrum occurs in the temperature region of the phase reversal (reflection minimum). This is attributed to the homodyne detection of the atomic signal, which in these conditions bears information about not only the dispersion but also the absorption of the atomic medium. Thus, selective reflection with a temperature-tunable window provides a tool, both very convenient and easy to implement, for studies of complex (absorptive and dispersive) response of an atomic vapor with a sub-Doppler resolution.

We have considered the application of the selective reflection using a temperature-tunable window for locking the laser diode frequency to the atomic resonance lines. The possibility to change the servo-lock point over a wide frequency range, $60-80 \mathrm{MHz}$ around the hyperfine resonance transitions and up to 600 $\mathrm{MHz}$ on the red wing of the line, by a simple change of the window temperature, is supported by numerical simulations performed for the conditions of the present experiment.

Acknowledgments. This work was supported, in part, by grant \#1323 of the Armenian Government. 
AVP thanks CNRS for the support of his stay at Ecole Normale Supérieure, where the main experiment was carried out. The authors are grateful to D. Bloch for stimulating discussions.

\section{References}

[1] R.W. Wood, Philos. Mag. 18, 187 (1909).

[2] J.L. Cojan, Ann. Phys. (Paris) 9, 385 (1954).

[3] J.P. Woerdman and M.F.H. Schuurmans, Opt. Commun. 14, 248 (1975).

[4] G. Nienhuis, F. Schuller and M. Ducloy, Phys. Rev. A 38, 5197 (1988).

[5] A.V. Papoyan, G.S. Sarkisyan and S.V. Shmavonyan, Opt. Spectrosc. 85, 649 (1998).

[6] V. Vuletić, V.A. Sautenkov, C. Zimmermann and T.W. Hänsch, Opt. Commun. 99, 185 (1993).

[7] A.V. Papoyan, J. Contemp. Phys. (Arm. Acad. Sci.) 33, 8 (1998).

[8] M. Chevrollier, M. Fichet, M. Oriá, G. Rahmat, D. Bloch and M. Ducloy, J. Phys. II (France) 2, 631 (1992).

[9] A. Weis, V.A. Sautenkov and T.W. Hänsch, Phys. Rev. A 45, 7991 (1992).

[10] B. Gross, N. Papageorgiou, V.A. Sautenkov and A. Weis, Phys. Rev. A 55, 2973 (1997).

[11] N. Papageorgiou, A. Weis, V.A. Sautenkov, D. Bloch and M. Ducloy, Appl. Phys. B 59, 123 (1994).

[12] R. Müller and A. Weis, Appl. Phys. B 66, 323 (1998).

[13] R.N. Li, S.T. Jia, D. Bloch and M. Ducloy, Opt. Commun. 146, 186 (1998).

[14] M.F.H. Schuurmans, J. Phys. (Paris) 37, 469 (1976).

[15] J. Guo, J. Cooper, A. Gallagher and M. Lewenstein, Opt. Commun. 110, 197 (1994).

[16] J. Guo, J. Cooper and A. Gallagher, Phys. Rev. A 53, 1130 (1996).

[17] M. Chevrollier, M. Oriá, J.G. de Souza, D. Bloch, M. Fichet and M. Ducloy, Phys. Rev. E 63, 046610 (2001).

[18] T.A. Vartanyan and F. Träger Opt. Commun. 110, 315 (1994); T.A. Vartanyan and D. Lin, Phys. Rev. A 51, 1959 (1995).

[19] E. Jahier, J. Guéna, Ph. Jacquier, M. Lintz, A.V. Papoyan and M.A. Bouchiat Appl. Phys. B 71, 561 (2000).

[20] G. Dutier, S. Saltiel, D. Bloch and M. Ducloy, J. Opt. Soc. Am. B 20, 793 (2003).

[21] D.A. Steck, Cs D line data, http://steck.us/alkalidata.

[22] J. J. Maki, M. S. Malcuit, J. E. Sipe, and R. W. Boyd, Phys. Rev. Lett. 67, 972 (1991). 
[23] M.A. Clifford, G.P.T. Lancaster, R.S. Conroy and K. Dholakia J. Modern Optics 47, 1933 (2000).

[24] V.V. Yashchuk, D. Budker and J.R. Davis, Rev. Sci. Instr. 71, 341 (2000).

[25] G. Wasik, W. Gawlik, J. Zachorowski and W. Zawadzki, Appl. Phys. B 75, 613 (2002). 

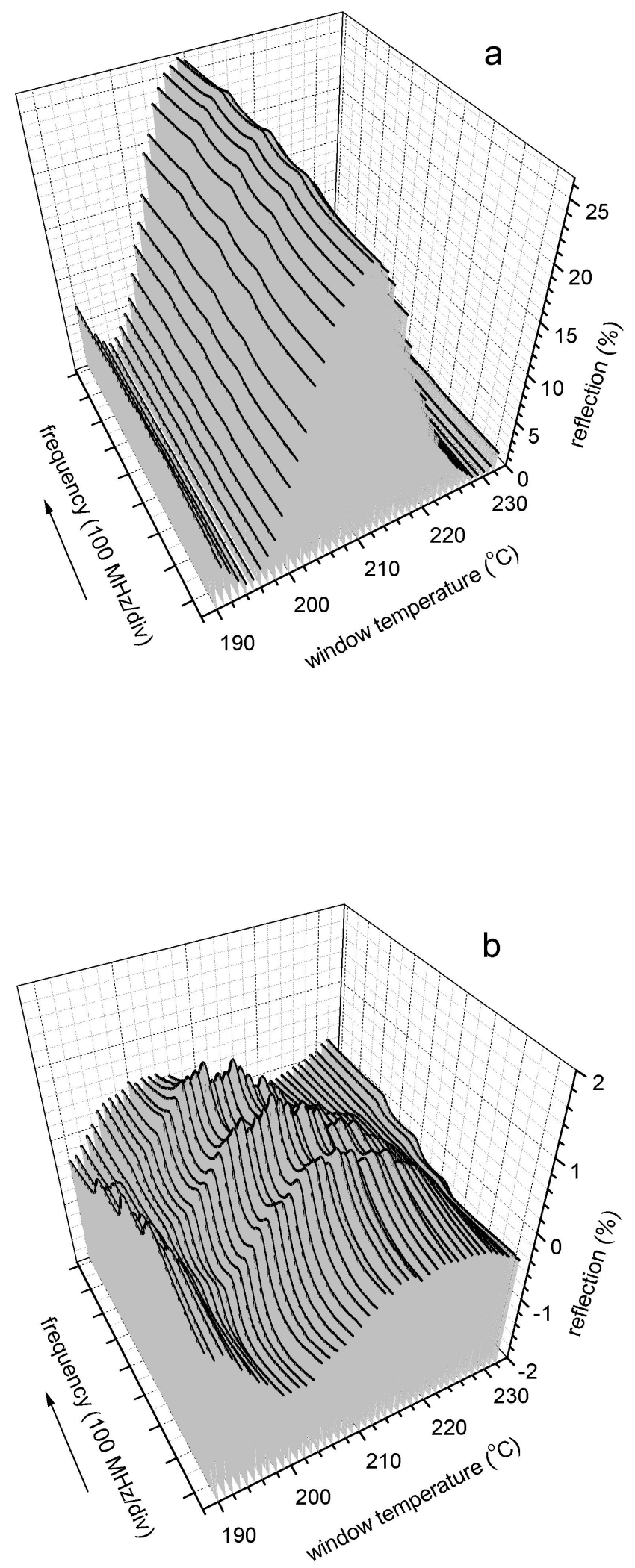


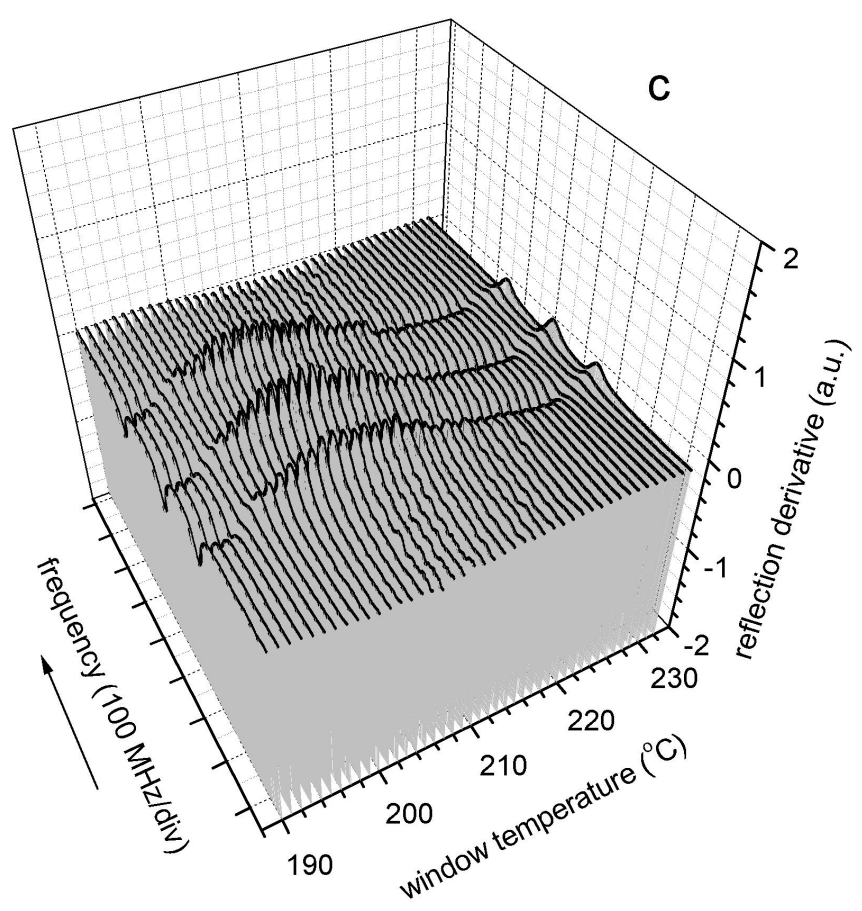

Figure 1: Results of measurements for $T_{s a}=160{ }^{\circ} \mathrm{C}$ and $T_{w}=190-232{ }^{\circ} \mathrm{C}$ : raw reflection spectra (a); same spectra with subtracted off-resonance contribution (see text) $(b)$; frequency derivative of the reflection spectra (c). The triple peak/dip structure corresponds to the $F_{g}=3 \rightarrow F_{e}=2,3,4$ hyperfine transitions of the $\mathrm{Cs}_{2}$ line. 


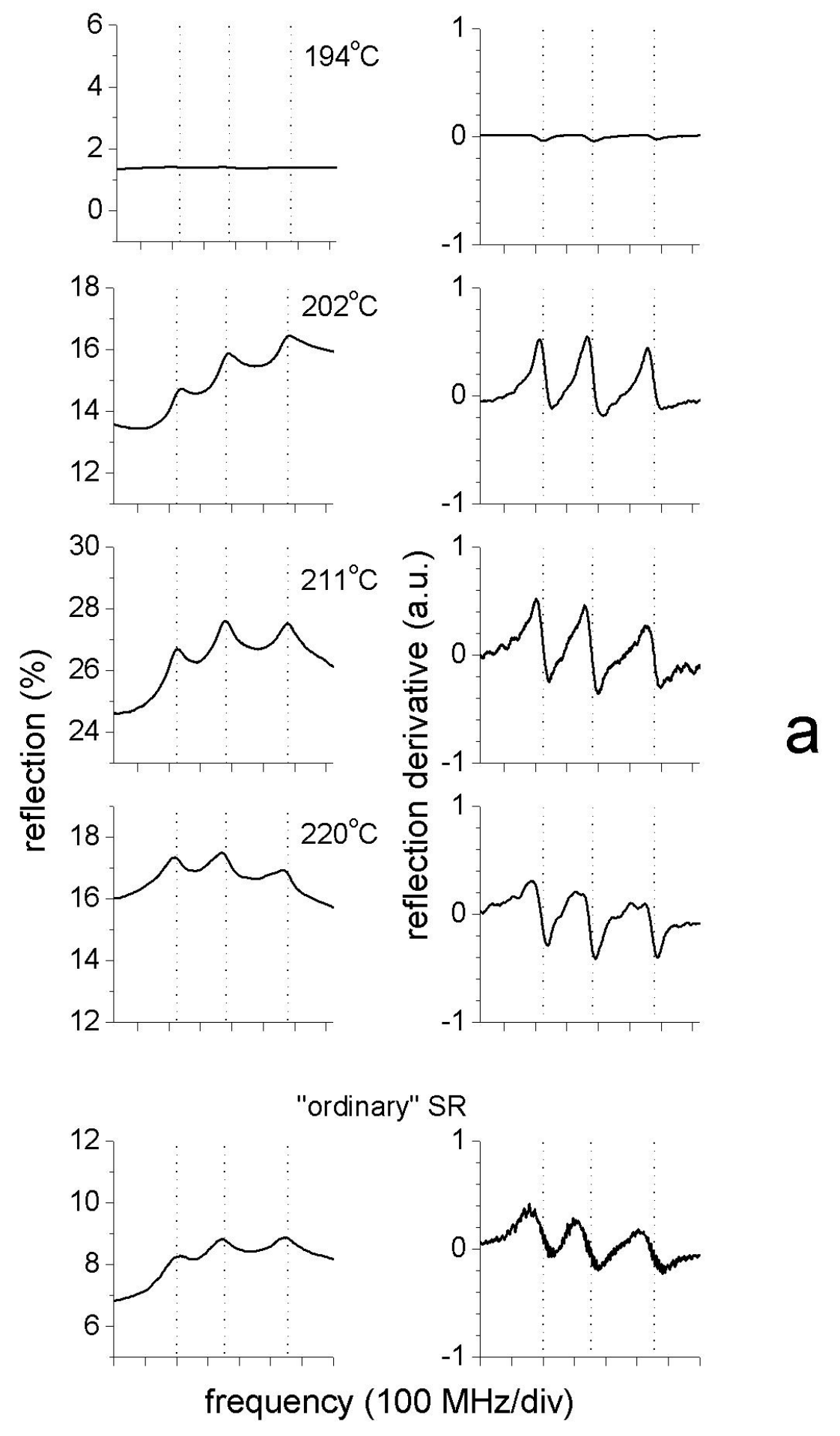




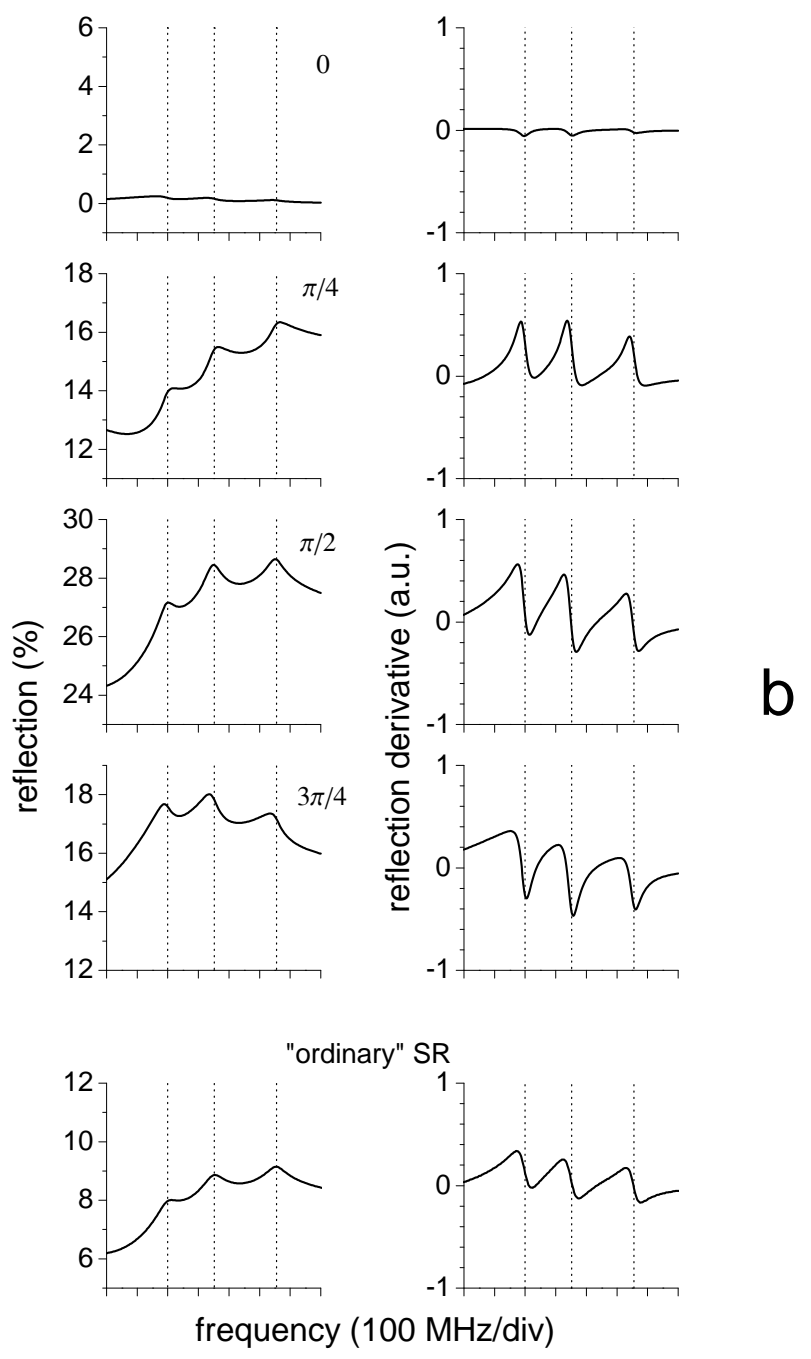

Figure 2: Results of measurements ( $a$ ) and numerical simulations $(b)$ of the selective reflection spectra (left columns) and their frequency derivatives (right columns) for $T_{s a}=160{ }^{\circ} \mathrm{C}$ and $T_{w}=194,202,211$, and $220{ }^{\circ} \mathrm{C}$ corresponding to $\varphi=0, \pi / 4, \pi / 2$, and $3 \pi / 4$ (four upper rows), and for the case of the wedged window cell with $T_{s a}=160{ }^{\circ} \mathrm{C}, T_{w}=208{ }^{\circ} \mathrm{C}$ (lower row). The positions of the $F_{g}=3 \rightarrow F_{e}=2,3,4$ hyperfine transitions of the $\mathrm{Cs}_{2} \mathrm{D}_{2}$ line are marked by the dotted lines. 


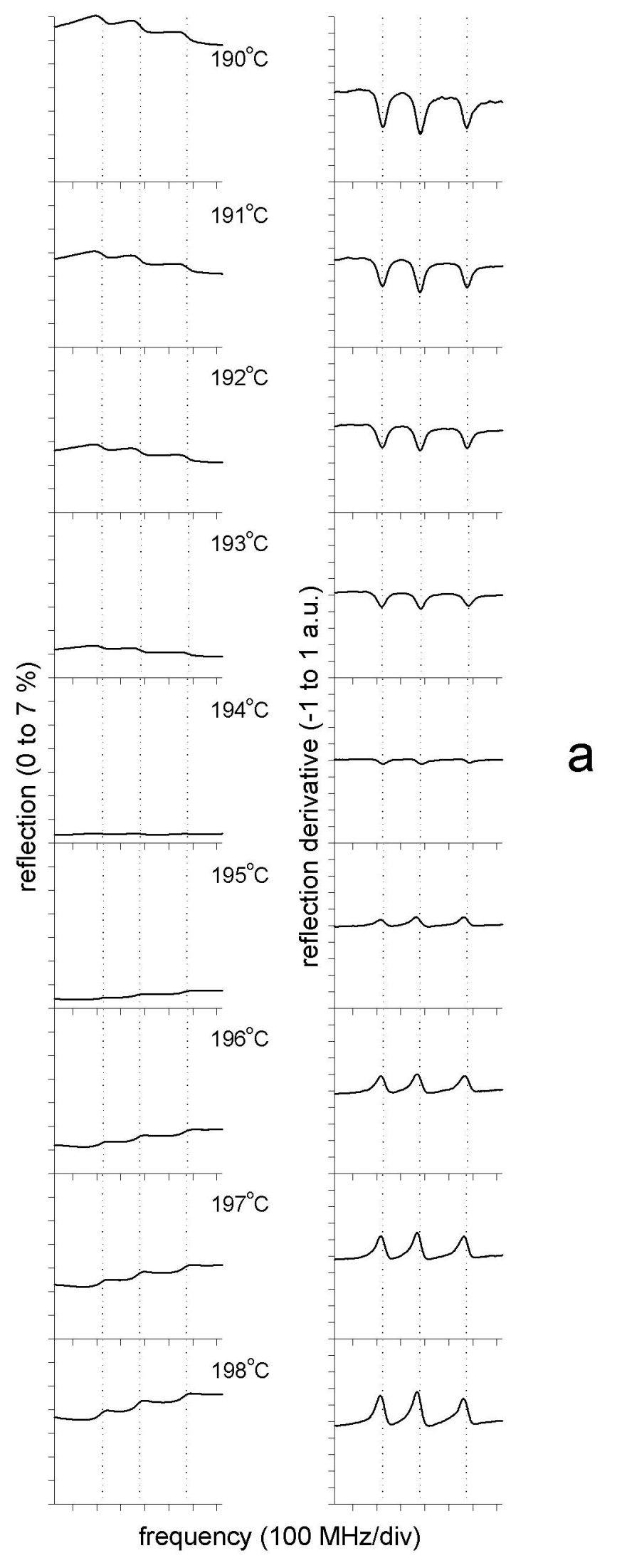




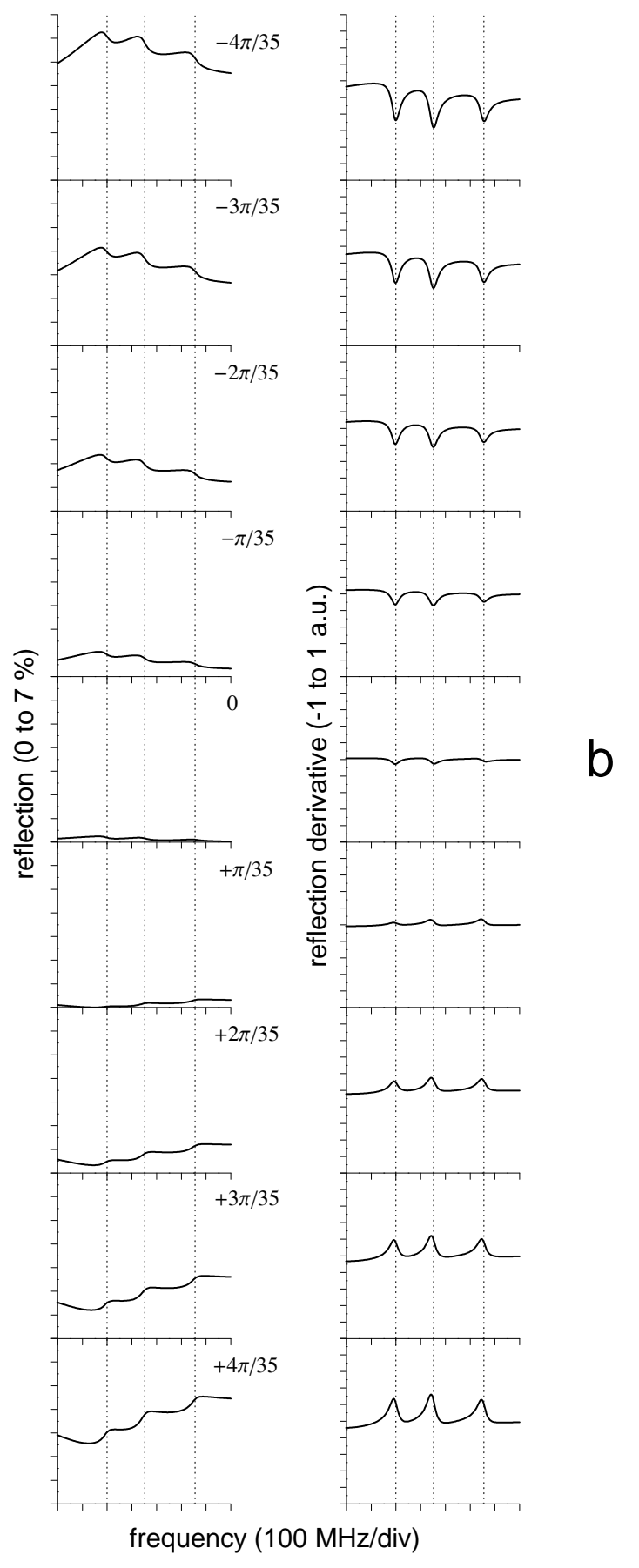

Figure 3: Results of measurements (a) and numerical simulations (b) for $T_{s a}=160{ }^{\circ} \mathrm{C}$ in the window temperature region of the reflection minimum. Left columns: reflection spectra; right columns: frequency derivative of the reflection spectra. The nine rows correspond to the indicated values of the window temperature $T_{w}$ (in $a$ ) and the phase $\varphi$ (in $b$ ); variation of $T_{w}$ by $1{ }^{\circ} \mathrm{C}$ corresponds to variation of $\varphi$ by $\pi / 35$. The positions of the $F_{g}=3 \rightarrow F_{e}=2,3,4$ hyperfine transitions of the $\mathrm{Cs}_{2} \mathrm{D}_{2}$ line are marked by the dotted lines. 


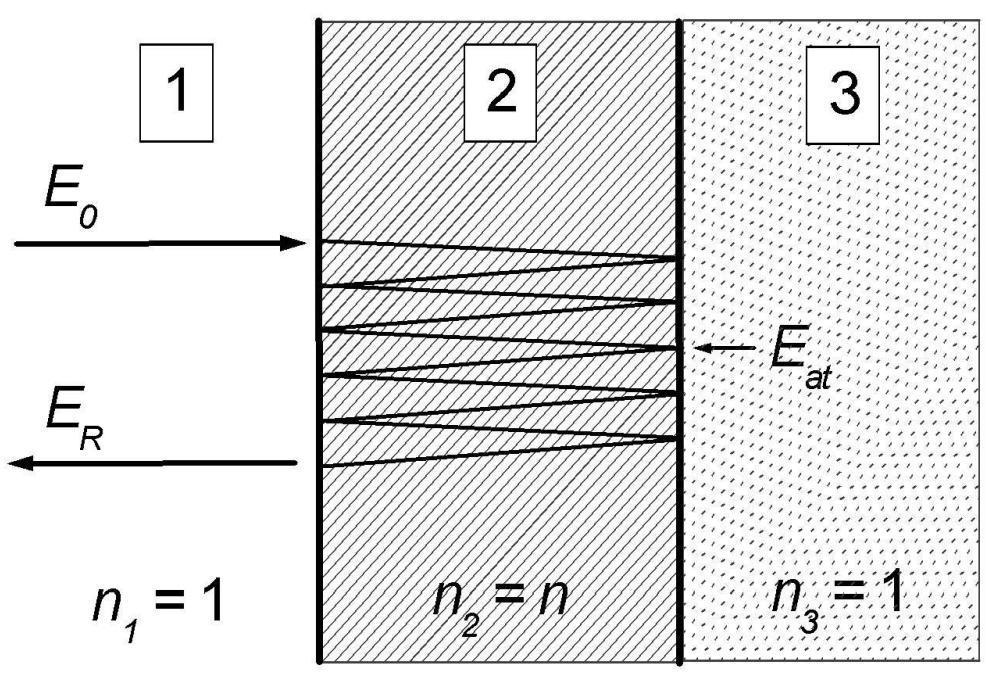

Figure 4: Reflection geometry. 1: air; 2: dielectric window with highly parallel faces; 3: atomic vapor. $E_{0}$ : incident laser radiation field; $E_{R}$ : total reflected radiation field; $E_{a t}$ : effective backward field generated in the atomic vapor (see text).
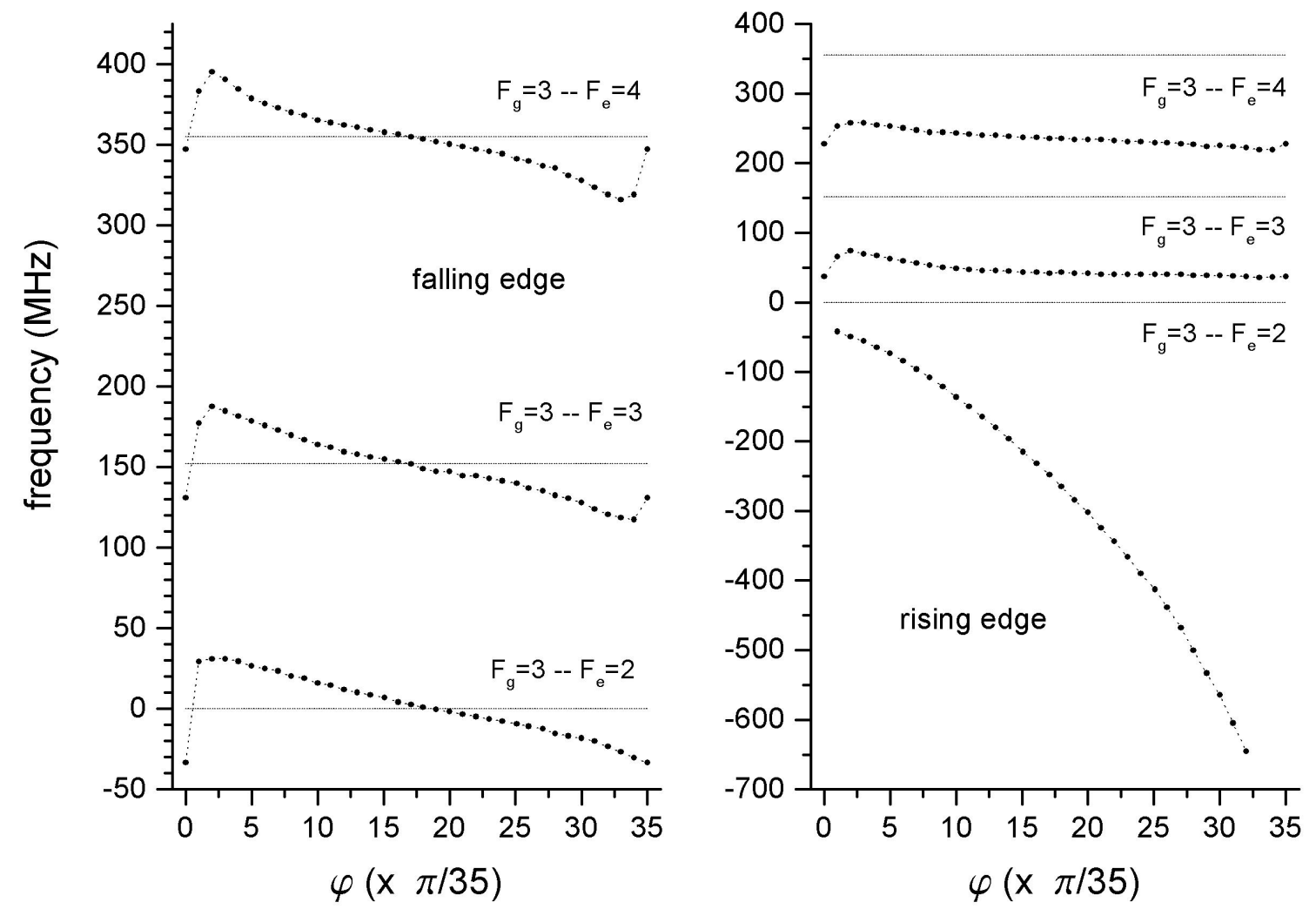

Figure 5: Calculated frequency of the zero-crossing points of the reflection derivative $v s$ the phase $\varphi$ on the falling (left column) and rising (right column) slopes. The horizontal dotted lines indicate the frequency positions of the hyperfine transitions. 